\title{
CS Research Square \\ Outcomes after Rotation Scarf with Akin Osteotomy for Severe Hallux Valgus
}

\section{Wenyong Xie}

Peking University People's Hospital

Hao Lu

Peking University People's Hospital

Guicheng Li

Peking University People's Hospital

Yusong Yuan

Peking University People's Hospital

Yijun Liu

Peking University People's Hospital

Hailin Xu ( $\nabla$ xuhailinfa@163.com )

Peking University People's Hospital

\section{Research Article}

Keywords: Hallux valgus, Rotation scarf osteotomy, Akin osteotomy, Outcomes

Posted Date: August 3rd, 2021

DOI: https://doi.org/10.21203/rs.3.rs-733306/v1

License: (c) (1) This work is licensed under a Creative Commons Attribution 4.0 International License.

Read Full License 


\section{Abstract}

Background: Hallux valgus is one of the most common foot deformities. Surgical treatment is the optimal choice for moderate to severe hallux valgus deformity. Rotation scarf with Akin osteotomy has become increasingly popular. The purpose of our study is to evaluate the long-term outcomes of rotation scarf with Akin osteotomy on severe hallux valgus.

Methods: We conducted a retrospective study of patients with hallux valgus who underwent surgery using rotation scarf with Akin osteotomy in our hospital between June 2014 and January 2020. Anteroposterior and lateral weightbearing $\mathrm{X}$-ray were performed before and after the operation. The parameters evaluated include (1) the hallux valgus angle (HVA), (2) intermetatarsal angle (IMA), (3) distal metatarsal articular angle (DMAA) and (4) tibial sesamoid position (TSP). A visual analog scale (VAS) was used to evaluate the pain level before and at the last follow-up after the operation. American Orthopaedic Foot \& Ankle Society (AOFAS) Forefoot Score were assessed before and at the last follow-up after the operation. Patient satisfaction assessment was also conducted at the time.

Results: A total of 42 patients with 50 feet met our inclusion criteria. Average age at the time of surgery was $61 \pm 9$ years. Follow-up data were obtained in all 42 patients. The average follow-up time was $47.4 \pm$ 20 months. All radiological parameteres including HVA, IMA, DMAA and TSP had statistically significant improvement $(p<0.001)$. The VAS score and AOFAS score was also statistically significant before operation and at the last follow-up after the operation $(p<0.001)$. There are $41(82 \%)$ feet in patients were very satisfied or satisfied.

Conclusion: Rotation scarf with Akin osteotomy is demonstrated to be safe, effective, and feasible for correcting severe hallux valgus. It can obtain good long-term correction with a low incidence of recurrence and metatarsalgia. Postoperative satisfaction and functional recovery of patients are significantly improved.

\section{Background}

Hallux valgus deformity is due to medial deviation of the first metatarsal and lateral deviation of the great toe, with or without coexisting pronation and subluxation of the metatarsophalangeal joint in a prevalence of $28.4 \%$ among adults.[1] The cause of deformity is complex and multifactorial, including internal and external risk factors. It has a higher prevalence in women and increases with age.[2] It may cause pain, deformities, inability to fit certain shoes, ulceration over the medial eminence and even impair daily activities.[3]

Surgical treatment is the optimal choice for moderate to severe hallux valgus deformity. It can relieve pain, correct the deformity, and improve function.[4, 5] More than 100 operative procedures have been described, with operative treatments varying from distal soft tissue releases to proximal and distal osteotomies.[5, 6] The scarf osteotomy was extensively used and reviewed.[7-9] In 1992, Duke et al. [10] modified the procedure surgical method and introduced the rotation scarf osteotomy. This osteotomy 
could maintain excellent stability and possibly greater correct the intermetatarsal angle (IMA),[11] which avoided postoperative complications and extended recovery time associated with more proximal osteotomies or arthrodesis.[12] Akin osteotomy is chosen by many doctors to correct phalanx deformity. Some authors regarded the Akin osteotomy mandatory for hallux valgus correction. $[13,14]$ In recent years, rotation scarf osteotomy with Akin osteotomy become increasingly popular.

Although there are many literatures about the surgical treatment of hallux valgus, rotation scarf with Akin osteotomy for severe hallux valgus has been little studied. The purpose of our study is to evaluate the long-term outcomes of rotation scarf with Akin osteotomy for severe hallux valgus.

\section{Methods}

This study was approved by the institutional review board of Peking University People's Hospital Ethics Review Committee. (2021PHB134-001) This is a retrospective review of a cohort of patients. The patients all gave their written consent to have all their outcome data, images and demographics. The all methods were performed in accordance with the relevant guidelines and regulations.[6, 10, 15-17]

\section{Inclusion and exclusion criteria}

We conducted a retrospective study of patients with hallux valgus who underwent surgery in our hospital between June 2014 and January 2020 with a single surgeon. The inclusion criteria for this study were (1) failed conservative treatment for 6 months, (2) hallux valgus angle (HVA) $\geq 40^{\circ}$ or IMA $\geq$ $20^{\circ}$ and (3) undergoing rotation scarf and Akin osteotomy. Patients with (1) ages $<18$, (2) first metatarsophalangeal arthritis, (3) surgical contraindications and (4) psychiatric disorders were excluded.

\section{Surgical technique}

Standard sterilization and draping techniques in the supine position with a tight pneumatic tourniquet were used for all patients in intraspinal or regional nerves anesthesia. We chose $1 \mathrm{~cm}$ distal to the first metatarsophalangeal joint over the hallux valgus and continuing proximally along the inferior margin of the first metatarsal, to the first metatarsocuneiform to make a $7 \mathrm{~cm}$ medial incision. A blunt dissection was conducted to avoid damage to the medial cutaneous nerve and the joint capsule was incised longitudinally. In all patients, the lateral soft-tissue release was performed through the medial incision through the dorsal skin, and the adductor hallucis tendon and lateral sesamoid ligament were released from their respective insertions in the metatarsal head and proximal phalanx (Figure 1A).

The medial eminence of the first metatarsal was resected at the sagittal groove. The long arm of the osteotomy line was conducted along the longitudinal axis of the metatarsal shaft, the greater the deformity, the longer the osteotomy line, and the osteotomy was performed horizontally on both sides of the osteotomy line, and the entire osteotomy line is in a " $Z$ " shape. The distal cut was on the dorsal aspect 
and the proximal cut was at approximately $45^{\circ}$ angles from the media proximal end to the lateral distal end in order to allow rotation to occur (Figure 1B). Then loosened the lateral capsule and rotated the inferior fragment toward the second metatarsal to reduce the IMA. Our goal was to reduce the IMA into normal range. One millimeter of rotation was equal to one degree of correction.[15] Then two 2.0 screws were fixed with $\mathrm{AO}$ technique. (Figure 1C). All patients underwent Akin osteotomy of the proximal phalanx. The Akin osteotomy was performed as a medial wedge and fixed by one 2.0 screw. (Figure $1 \mathrm{C}$, D). Then the capsule was closed with 2-0 absorbable suture, and skin was closed subcuticularly using 4-0 silk suture.

\section{Postoperative protocol}

All patients completed the postoperative program. Wrap the hallux correction position with a crepe bandage. Two weeks after the operation, the suture was removed. The offloading orthosis in a rigid insole was used to carry weight 6 weeks later. After 12 weeks, patients were allowed to wear normal shoes and walk normally. It was allowed to return to sports after 6 months.

\section{Outcome measures}

Anteroposterior and lateral weightbearing X-ray were performed before and after the operation. Radiographs were analyzed in a digital manner using the Picture Archiving and Communication Systems (PACS). The parameters evaluated include (1) the HVA, (2) IMA, (3) distal metatarsal articular angle (DMAA) and (4) tibial sesamoid position (TSP) in relation to the midshaft axis of the first metatarsal (7part grading system).[16] A visual analog scale (VAS) (ranging from 1 to 10) was used to evaluate the pain level before and at the last follow-up after the operation. American Orthopaedic Foot \& Ankle Society (AOFAS) Forefoot Score were assessed before and at the last follow-up after the operation. Patient satisfaction was analyzed though answering the question "Are you satisfied with the result of the surgery?" with 1 of 4 responses: (1) very satisfied, (2) satisfied, (3) undecided, or (4) not satisfied.[17]

\section{Statistical analysis}

Statistical analysis was performed using SPSS 23.0 (SPSS, Chicago, IL, USA). The continuous variables were described as median (interquartile range) or mean \pm standard deviation for quantitative data and as numbers for categorical measures. Continuous normally distributed data were analyzed using the paired Student $t$ test. Continuous nonnormally distributed paired data were analyzed using the Wilcoxon ranksum test. $P<0.05$ was considered significant.

\section{Results}


A total of 42 patients with 50 feet met our inclusion criteria, including 29 on the right and 21 on the left. There were only 3 male patients. Average age at the time of surgery was $61 \pm 9$ years. Follow-up data were obtained in all 42 patients. The average follow-up time was $47.4 \pm 20$ months. (Table 1 ) All patients had substantial foot pain during daily life or sports activity before the operation. The VAS score and AOFAS score before operation and at the last follow-up after the operation were showed in Table 2 and the difference between the preoperative and postoperative stage was statistically significant $(P<0.001)$ (Fig. 2).

Table 1

Patient demographics

\begin{tabular}{|ll|}
\hline & Number (\%) \\
\hline Number of feet & 50 \\
\hline Right feet & $21(42)$ \\
\hline Left feet & $13(26)$ \\
\hline Bilateral & $16(32)$ \\
\hline Age at time of surgery (years) & $61 \pm 9$ \\
\hline Female & $47(94)$ \\
\hline Male & $3(6)$ \\
\hline Follow-up time (months) & $47.4 \pm 20$ \\
\hline
\end{tabular}


Table 2

Preoperative and postoperative radiographic and clinical results.

\begin{tabular}{|lllll|}
\hline & Preoperative & Postoperative & Mean correction & P value \\
\hline HVA & $45.7^{\circ} \pm 5.0^{\circ}$ & $12.1^{\circ} \pm 7.2^{\circ}$ & $33.6^{\circ}$ & $<0.001^{\mathrm{a}}$ \\
\hline IMA & $15.2^{\circ} \pm 3.4^{\circ}$ & $4.2^{\circ} \pm 2.5^{\circ}$ & $11^{\circ}$ & $<0.001^{\mathrm{a}}$ \\
\hline DMAA & $29.7^{\circ} \pm 11.8^{\circ}$ & $16.0^{\circ} \pm 7.1^{\circ}$ & $13.7^{\circ}$ & $<0.001^{\mathrm{a}}$ \\
\hline TSP & $6(5-6)$ & 2 & $<0.001^{\mathrm{b}}$ \\
\hline VAS & $5(4-5)$ & 5 & $<0.001^{\mathrm{b}}$ \\
\hline AOFAS & $35(32-40)$ & 60 & \\
\hline $\begin{array}{l}\text { IM intermetatarsal, HV hallux valgus, TSP tibial sesamoid position, VAS visual analog scale, AOFAS } \\
\text { American Orthopaedic Foot and Ankle Society. }\end{array}$ & & \\
\hline $\begin{array}{l}\text { a, Values are given as the mean } \pm \text { standard deviation, the statistical analysis was conducted using } \\
\text { paired Student t test. }\end{array}$ & $9(0-1)$ & \\
\hline $\begin{array}{l}\text { b, Values are given as the median (interquartile range), the statistical analysis was conducted using a } \\
\text { Wilcoxon rank-sum test. }\end{array}$
\end{tabular}

The HVA decreased from $45.7^{\circ} \pm 5.0^{\circ}$ before operation to $12.1^{\circ} \pm 7.2^{\circ}(\mathrm{P}<0.001)$ after operation.

Postoperative IMA reached $4.2^{\circ} \pm 2.5^{\circ}$ from $15.2^{\circ} \pm 3.4^{\circ}$ preoperatively $(P<0.001)$. The DMAA decreased from $29.7^{\circ} \pm 11.8^{\circ}$ before operation to $16.0^{\circ} \pm 7.1^{\circ}(\mathrm{P}<0.001)$ after operation, which was close to the normal range. Postoperative TSP reached 4 from 6 preoperatively (Table 2) (Fig. 3). Regarding the overall degree of satisfaction with the treatment, 20 (40\%) feet in patients were very satisfied (score 1), 21(42\%) feet in were satisfied (score 2), 6(12\%) feet in were undecided (score 3) and 3(6\%) feet in were not satisfied (score 4).

Complications occurred in 8 feet (16\%). The recurrence of hallux valgus was defined as an HVA $\geq 20^{\circ}$.[18] Recurrence was found in $6(12 \%)$ of the 50 feet at the time of the last follow-up. Among the 6 recurrent feet, one patient had superficial wound infections, which were treated with oral antibiotics and local wound care, and two patients experienced pain caused by metastatic metatarsalgia. In the remaining 44 feet, one patient experienced pain caused by metastatic metatarsalgia and one patient developed hallux varus with metatarsal pain after operation and was treated conservatively. The metastatic metatarsalgia can be relieved by taking modified shoes or a brace. Table 3 showed the relationship between patient satisfaction and complications. None of the patients had resorption below the articular surface, bone sclerosis, and necrosis and nonunion. 
Table 3

Patient satisfaction

\begin{tabular}{|lll|}
\hline Patient satisfaction & Number (\%) & Complication (Number) \\
\hline Very satisfied & $20(40)$ & Hallux varus (1), Recurrence (2) \\
\hline Satisfied & $21(42)$ & Recurrence (1) \\
\hline undecided & $6(12)$ & Recurrence with infections (1) \\
\hline Not satisfied & $3(6)$ & Recurrence with metatarsalgia (2) and Metatarsalgia (1) \\
\hline
\end{tabular}

\section{Discussion}

Our study illustrates that the rotation scarf with Akin osteotomy for severe hallux valgus was good in terms of treatment outcomes and patient satisfaction. Scarf osteotomy was conducted in the treatment of moderate and severe hallux valgus. However, clinical studies were required to further investigate its effects on severe hallux valgus. It was also indispensable for severe hallux valgus to conducted soft tissue release. In this study, all patients underwent rotation scarf with Akin osteotomy combined with soft tissue release, and the efficacy of the treatment was certain. The preoperative AOFAS score was 35 and patients were with moderate to severe pain with significant deformity. The average age was 61 with a gender preponderance of females. Those were consistent with the other studies. $[9,19]$

Rotation scarf osteotomy was a Z-shaped diaphyseal osteotomy of the first metatarsal, which mainly completed deformity correction through translation and rotation.[20] During the operation, we always try to maintain the length of the first metatarsal and displace the metatarsal head in a plantarly direction as part of the rotation scarf osteotomy to reduce the possibility of metastatic metatarsalgia. In this study, three patients had mild metastatic metatarsalgia. Although 6 patients occurred recurrence of hallux valgus, the average HVA of recurrence of hallux valgus is $25.7^{\circ}$, which was close to the normal reported range of $15^{\circ}$.[21] Rotation scarf osteotomy had a better effect on the correction of IMA, and it also had a certain correction for DMAA.[20] Akin osteotomy combined with the first metatarsal osteotomy was increasingly acknowledged in the literature.[7, 9] Through evaluating the treatment of severe hallux valgus with rotation scarf with Akin osteotomy we found that comparing to osteotomy alone or other proximal metatarsal osteotomy, rotation scarf with Akin osteotomy had many advantages, of which included: (1) Accurate correction of the IMA, one millimeter lateral displacement of the plantar head is equal to one degree of IMA correction, which can be evaluated and corrected intraoperatively.[15] (2) A large area of bone-to-bone contact reduces the risk of nonunion, and metatarsal shaft osteotomy reduces the necrosis of the metatarsal head. (3) The high stability of the osteotomy, inferior and superior fragments of the metatarsal being pushed together by weight bearing forces rather than being distracted apart by those forces. (4) Rotation of the inferior fragment can also make the IMA greater correction, which is greater than what can be achieved by other osteotomy. (5) Akin osteotomy can effectively correct the enlargement of DMAA, and effectively assist metatarsal osteotomy, which significantly 
improves the efficacy and satisfaction rate and fully compensates for the deficiencies exhibited by rotation scarf osteotomy. [22]

Rotation scarf osteotomy was among the most common operative procedures for hallux valgus deformity. Common complications of rotation scarf osteotomy include first metatarsal fracture, metastatic metatarsalgia, infection, medial dorsal cutaneous nerve injury of the hallux, and recurrence of deformity. $[8,23,24]$ Among them, metastatic metatarsalgia was a serious impact on patient satisfaction and three not satisfied patients all developed metastatic metatarsalgia in our study. Smith et al.[24] reported that, based on early experience, there was a $6 \%$ perioperative complication for those beginning to master the scarf osteotomy. Before modifying this osteotomy, Coetzee et al. [8] reported a complication rate of $47 \%$. In our study, it was found that the incidence of complications was relatively rare. The incidence of complications can be reduced when surgeon was skilled in this osteotomy.

Hallux valgus deformity was often accompanied by enlarged DMAA. One of the reasons for recurrence deformity was that DMAA had not been well corrected.[25, 26] During the rotation scarf osteotomy, DMAA can be corrected to a certain extent through pushing the inferior fragment to lateral and rotating the inferior fragment. Then the match of metatarsophalangeal can be restored, and the rate of recurrence deformity can be reduced. In our study, DMAA decreased from $29.7^{\circ} \pm 11.8^{\circ}$ before surgery to $16.0^{\circ} \pm 7.1^{\circ}$ after surgery. It should be noted that although DMAA has decreased after surgery, it has not returned to a completely normal state. The main effect of rotation scarf osteotomy is reflected in the recovery of HVA and IMA, and the corrective effect of DMAA is limited. On the other hand, rotation scarf with Akin osteotomy is also useful for the restoration of TSP which is associated with the prognosis of postoperative outcomes.[18]

In AOFAS and VAS scores, there was a significant difference before and after operation. And the postoperative AOFAS score increased by 60 . The VAS score was also reduced to 0 after the operation. Jeuken RM et al.[27] found even after a prolonged follow-up, scarf osteotomy scored around 32 points higher than preoperative measures in AOFAS. Perugia D et al.[9] studied scarf osteotomy for severe hallux valgus and the results were generally good and the mean correction was 54.1 in AOFAS.

Our study does have some limitations. Firstly, present study is a retrospective without control group designed study. It cannot compare the advantages and disadvantages of rotation scarf with Akin osteotomy. Secondly, although the sample size of severe hallux valgus is relatively large, it is still necessary to increase the sample size for further investigation. Therefore, a larger sample of long-term follow-up and randomized controlled clinical trials are needed.

\section{Conclusions}

In conclusion, rotation scarf with Akin osteotomy is demonstrated to be safe, effective, and feasible for correcting severe hallux valgus. It can obtain good long-term correction with a low incidence of recurrence and metatarsalgia. Postoperative satisfaction and functional recovery of patients are significantly 
improved. However, further studies with large sample sizes and long follow-up duration need to be done for getting more information about long-term consequences of this surgical procedure.

\section{Abbreviations}

HVA: hallux valgus angle; IMA: intermetatarsal angle; DMAA: distal metatarsal articular angle; TSP: tibial sesamoid position; VAS: A visual analog scale; AOFAS: American Orthopaedic Foot \& Ankle Society Forefoot Score; PACS: Picture Archiving and Communication Systems

\section{Declarations}

\section{Ethics approval and consent to participate}

This study was approved by the institutional review board of our institution (Peking University People's Hospital Ethics Review Committee, 2021PHB134-001). This is a retrospective review of a cohort of patients. All patients had undergone routine diagnosis and were treated with routine surgery. The patients all gave their written consent to have all their outcome data, images and demographics.

\section{Consent for publication}

The patients (all adults) all gave their explicit written consent to have all their outcome data, images and demographics included in this review writeup and for publication.

\section{Availability of data and materials}

The datasets and materials are available from corresponding authors on reasonable request.

\section{Competing interests}

The authors declare that there is no conflict of interest.

\section{Funding}

1. Capital's Funds for Health Improvement and Research (Number 2020-2-4086). 2. The Beijing Municipal Science and Technology Project, Z181100001718159. 3. Key Laboratory of Trauma and Neural Regeneration (Peking University), Ministry of Education.

\section{Authors' contributions}


WYX, HL and GCL performed data analysis, as well as writing the manuscript. YJL and YSY contributed to the collection of data and manuscript editing. HLX edited the manuscript and were the guarantor of this article.

\section{Acknowledgements}

We thank BYZ and YQ for their support, which is essential for this work.

\section{For methods}

The authors confirm that all methods were performed in accordance with the relevant guidelines and regulations.

\section{References}

1. Fakoor M, Sarafan N, Mohammadhoseini P, Khorami M, Arti H, Mosavi S, Aghaeeaghdam A: Comparison of Clinical Outcomes of Scarf and Chevron Osteotomies and the McBride Procedure in the Treatment of Hallux Valgus Deformity. The archives of bone and joint surgery 2014, 2(1):31-36.

2. Nix $S$, Smith M, Vicenzino B: Prevalence of hallux valgus in the general population: a systematic review and meta-analysis. Journal of foot and ankle research 2010, 3:21.

3. Iselin LD, Munt J, Symeonidis PD, Klammer G, Chehade M, Stavrou P: Operative management of common forefoot deformities: a representative survey of Australian orthopaedic surgeons. Foot \& ankle specialist 2012, 5(3):188-194.

4. Stanekova K, Assal M, Crevoisier X: [Evidence based treatment of hallux valgus]. Revue medicale suisse 2017, 13(587):2158-2163.

5. Fraissler $\mathrm{L}$, Konrads $\mathrm{C}$, Hoberg $\mathrm{M}$, Rudert $\mathrm{M}$, Walcher $\mathrm{M}$ : Treatment of hallux valgus deformity. EFORT open reviews 2016, 1(8):295-302.

6. Zirngibl B, Grifka J, Baier C, Götz J: [Hallux valgus: Etiology, diagnosis, and therapeutic principles]. Der Orthopade 2017, 46(3):283-296.

7. Jones S, Al Hussainy HA, Ali F, Betts RP, Flowers MJ: Scarf osteotomy for hallux valgus. A prospective clinical and pedobarographic study. The Journal of bone and joint surgery British volume 2004, 86(6):830-836.

8. Coetzee JC: Scarf osteotomy for hallux valgus repair: the dark side. Foot Ankle Int 2003, 24(1):2933.

9. Perugia D, Basile A, Gensini A, Stopponi M, Simeonibus AU: The scarf osteotomy for severe hallux valgus. Int Orthop 2003, 27(2):103-106.

10. Duke HF: Rotational scarf (Z) osteotomy bunionectomy for correction of high intermetatarsal angles. Journal of the American Podiatric Medical Association 1992, 82(7):352-360. 
11. Deenik AR, Pilot P, Brandt SE, van Mameren H, Geesink RG, Draijer WF: Scarf versus chevron osteotomy in hallux valgus: a randomized controlled trial in 96 patients. Foot Ankle Int 2007, 28(5):537-541.

12. Smith SE, Landorf KB, Butterworth PA, Menz HB: Scarf versus chevron osteotomy for the correction of 1-2 intermetatarsal angle in hallux valgus: a systematic review and meta-analysis. $J$ Foot Ankle Surg 2012, 51(4):437-444.

13. Larholt J, Kilmartin TE: Rotational scarf and akin osteotomy for correction of hallux valgus associated with metatarsus adductus. Foot Ankle Int 2010, 31(3):220-228.

14. Lai MC, Rikhraj IS, Woo YL, Yeo W, Ng YCS, Koo K: Clinical and Radiological Outcomes Comparing Percutaneous Chevron-Akin Osteotomies vs Open Scarf-Akin Osteotomies for Hallux Valgus. Foot Ankle Int 2018, 39(3):311-317.

15. Sarrafian SK: A method of predicting the degree of functional correction of the metatarsus primus varus with a distal lateral displacement osteotomy in hallux valgus. Foot \& ankle 1985, 5(6):322326.

16. Hardy RH, Clapham JC: Observations on hallux valgus; based on a controlled series. The Journal of bone and joint surgery British volume 1951, 33-b(3):376-391.

17. Kaufmann G, Mortlbauer L, Hofer-Picout P, Dammerer D, Ban M, Liebensteiner M: Five-Year Follow-up of Minimally Invasive Distal Metatarsal Chevron Osteotomy in Comparison with the Open Technique: A Randomized Controlled Trial. J Bone Joint Surg Am 2020, 102(10):873-879.

18. Park CH, Lee WC: Recurrence of Hallux Valgus Can Be Predicted from Immediate Postoperative NonWeight-Bearing Radiographs. J Bone Joint Surg Am 2017, 99(14):1190-1197.

19. Kilmartin TE, O'Kane C: Combined rotation scarf and Akin osteotomies for hallux valgus: a patient focussed 9 year follow up of 50 patients. Journal of foot and ankle research 2010, 3:2.

20. Peng J, Wang Q, Jha AJ, Pitts C, Li Q, Brahmbhatt A, Shah AB: The Rotational Effect of Scarf Osteotomy With Transarticular Lateral Release on Hallux Valgus Correction. J Foot Ankle Surg 2021, 60(2):328-332.

21. Law GW, Tay KS, Lim JWS, Zhang KT, Seng C, Yeo NEM, Koo K, Rikhraj IS: Effect of Severity of Deformity on Clinical Outcomes of Scarf Osteotomies. Foot Ankle Int 2020, 41(6):705-713.

22. Kaufmann G, Hofmann M, Ulmer H, Putzer D, Hofer P, Dammerer D: Outcomes after scarf osteotomy with and without Akin osteotomy a retrospective comparative study. J Orthop Surg Res 2019, 14(1):193.

23. Kristen KH, Berger C, Stelzig S, Thalhammer E, Posch M, Engel A: The SCARF osteotomy for the correction of hallux valgus deformities. Foot Ankle Int 2002, 23(3):221-229.

24. Smith AM, Alwan T, Davies MS: Perioperative complications of the Scarf osteotomy. Foot Ankle Int 2003, 24(3):222-227.

25. Şahin N, Cansabuncu G, Çevik N, Türker O, Özkaya G, Özkan Y: A randomized comparison of the proximal crescentic osteotomy and rotational scarf osteotomy in the treatment of hallux valgus. Acta orthopaedica et traumatologica turcica 2018, 52(4):261-266. 
26. Raikin SM, Miller AG, Daniel J: Recurrence of hallux valgus: a review. Foot Ankle Clin 2014, 19(2):259-274.

27. Jeuken RM, Schotanus MG, Kort NP, Deenik A, Jong B, Hendrickx RP: Long-term Follow-up of a Randomized Controlled Trial Comparing Scarf to Chevron Osteotomy in Hallux Valgus Correction. Foot Ankle Int 2016, 37(7):687-695.

\section{Figures}
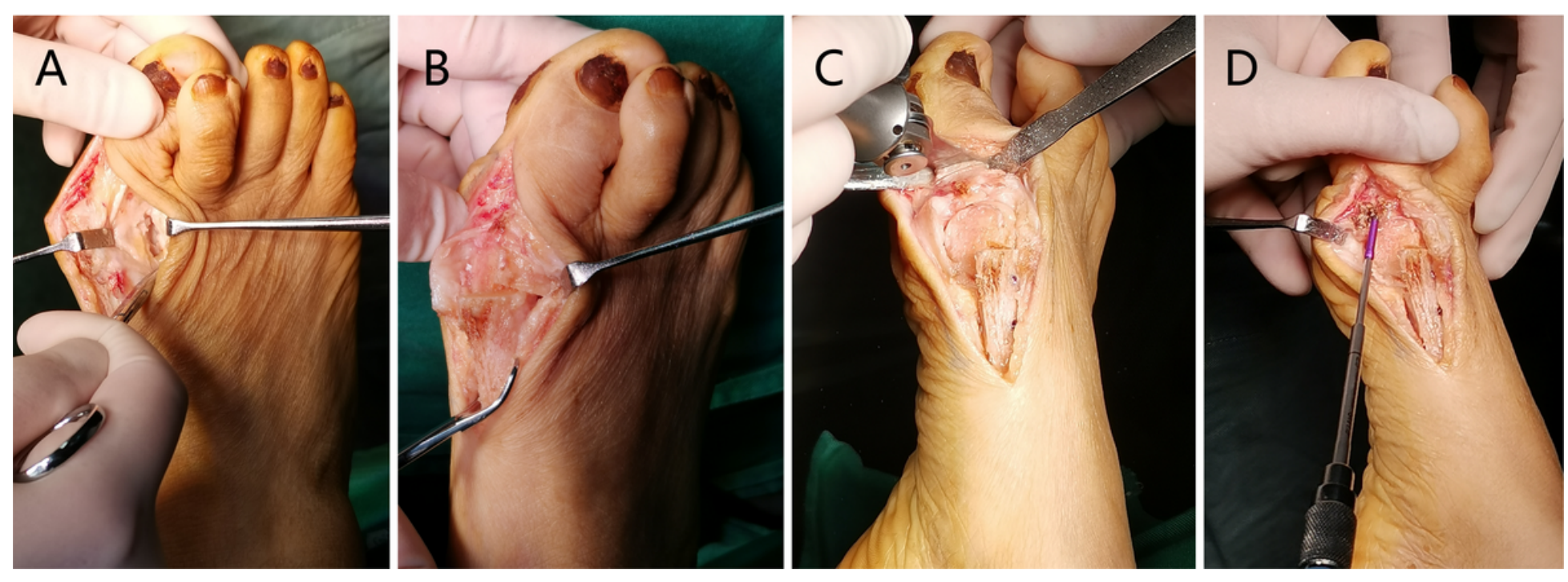

Figure 1

Intraoperative view of rotation scarf with Akin osteotomy. A Surgical view of lateral soft tissue release. B Scarf osteotomy after rotation and an adequate lateral moving. C The scarf osteotomy was fixed with two 2.0 cortical screws and Akin osteotomy was conducting. D The Akin osteotomy was fixed by one 2.0 cortical screw. 

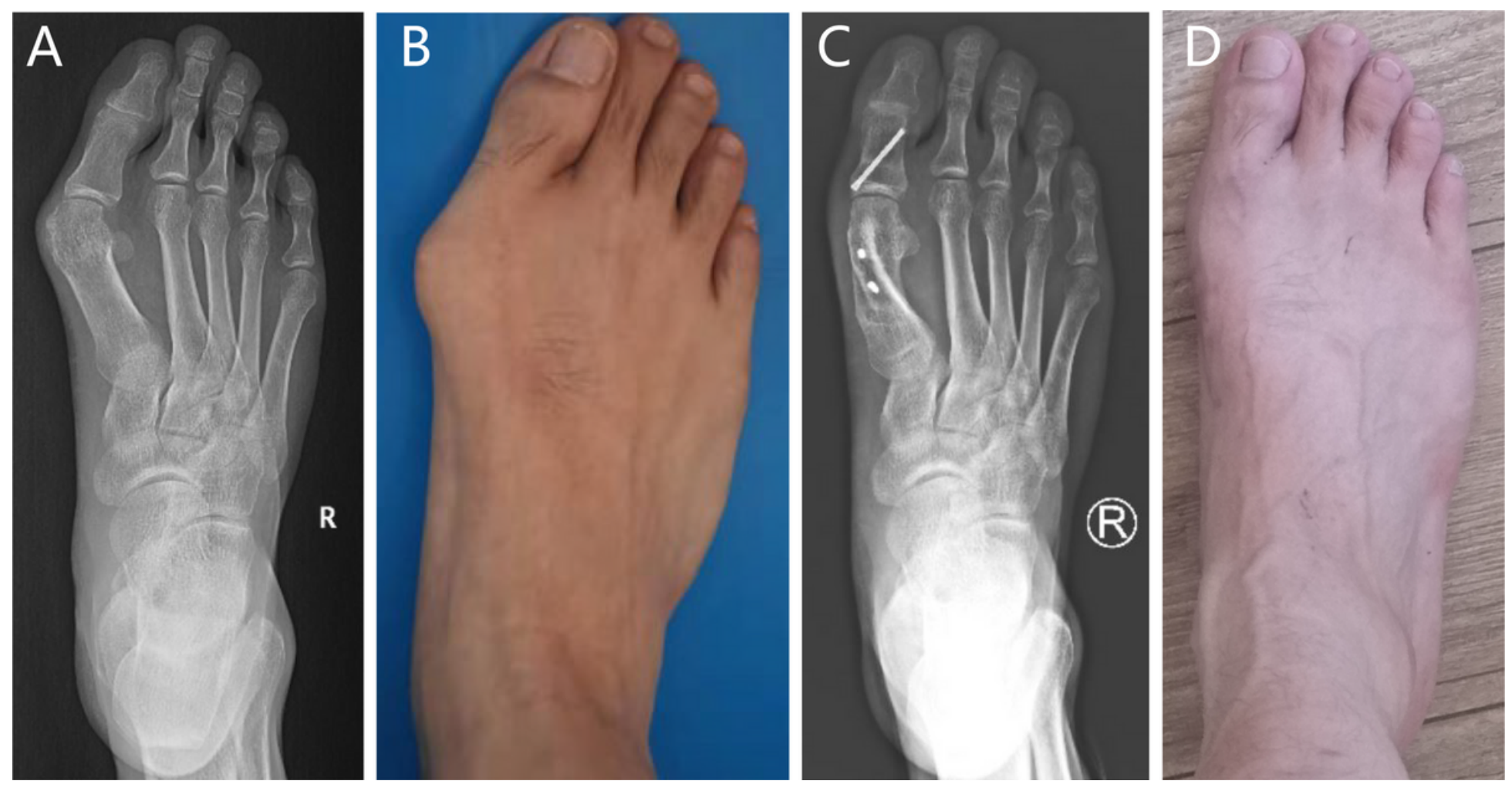

\section{Figure 2}

Preoperative (A, B) and follow-up (C, D) of a severe hallux valgus (HV) deformity corrected with rotation scarf with Akin osteotomy. 


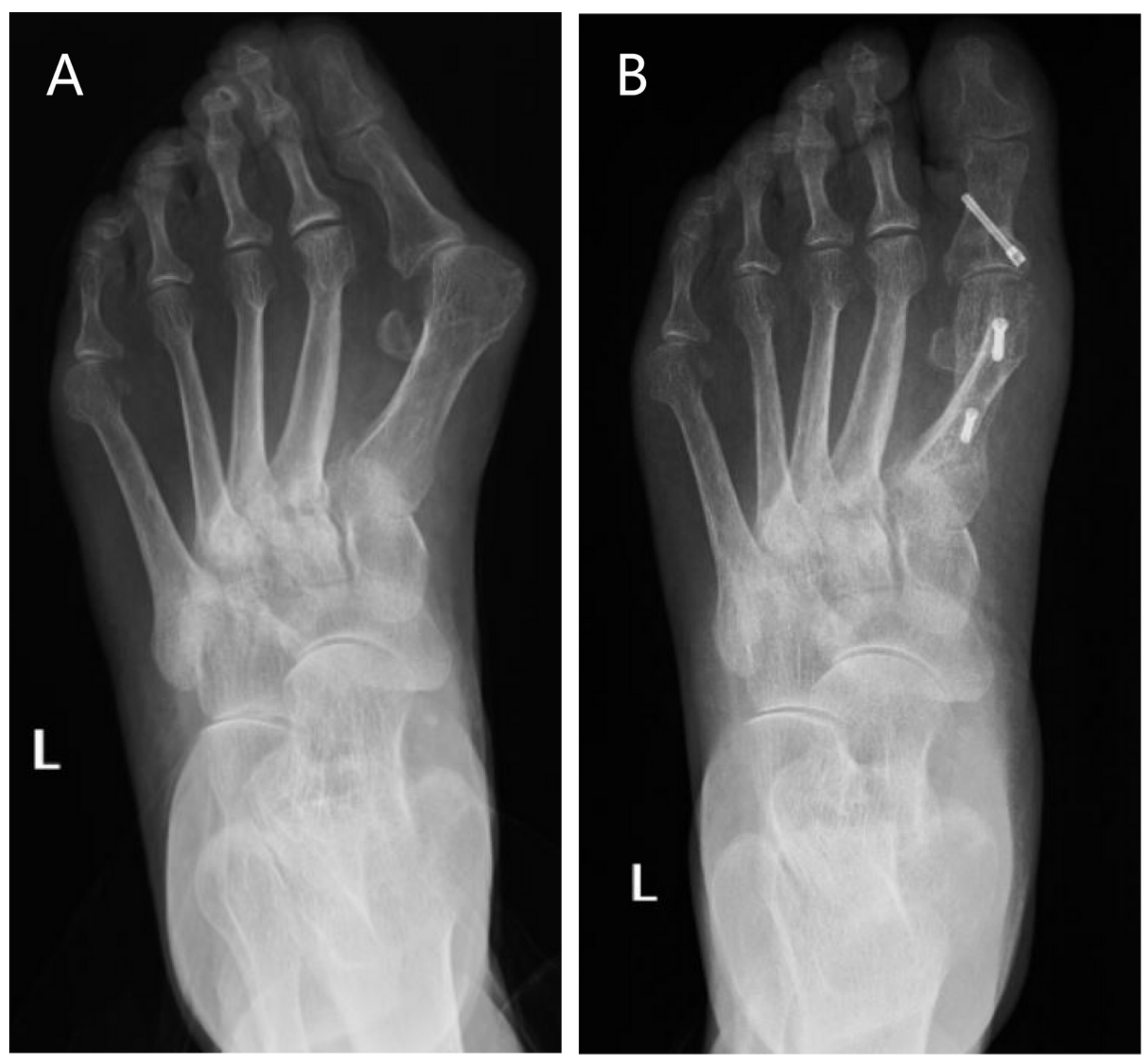

\section{Figure 3}

Preoperative dorsoplantar standing radiograph (A) and Follow-up dorsoplantar standing radiograph (B) of an extremely severe hallux valgus (HV) deformity corrected with scarf with Akin osteotomy showing the correction of the intermetatarsal angle, $\mathrm{HV}$ angle, distal metatarsal articular angle and tibial sesamoid position. 